On the Convergence of Interior-Point Methods to the Center of the Solution Set in Linear Programming

Yin Zhang and

R.A. Tapia

September, 1991

TR91-30 



\title{
On the Convergence of Interior-Point Methods to the Center of the Solution Set in Linear Programming
}

\author{
Yin Zhang *and Richard A. Tapia ${ }^{\dagger}$
}

September, 1991

\begin{abstract}
The notion of the central path plays an important role in the convergence analysis of interior-point methods. Many interior-point algorithms have been developed based on the principle of following the central path, either closely or otherwise. However, whether such algorithms actually converge to the center of the solution set has remained an open question. In this paper, we demonstrate that under mild conditions, when the iteration sequence generated by a primal-dual interior-point method converges, it converges to the center of the solution set.
\end{abstract}

Keywords: Linear programming, Primal-dual interior-point algorithms, Central path, Center of the solution set.

Abbreviated Title: On Convergence to the Center

*Department of Mathematics and Statistics and visiting member of the Center for Research in Parallel Computation, University of Maryland, Baltimore County Campus, Baltimore, Maryland 21228. This author was supported in part by NSF DMS-9102761 and DOE DE-FG05-91ER25100.

${ }^{\dagger}$ Department of Mathematical Sciences and the Center for Research in Parallel Computation, Rice University, Houston, Texas 77251-1892. This author was supported in part by NSF Coop. Agr. No. CCR-8809615, AFOSR 89-0363, DOE DEFG05-86ER25017 and ARO 9DAAL03-90-G-0093. 



\section{Introduction}

We consider linear programs in the standard form:

$$
\begin{array}{cl}
\operatorname{minimize} & c^{T} x \\
\text { subject to } & A x=b \\
& x \geq 0
\end{array}
$$

where $c, x \in \mathbf{R}^{n}, b \in \mathbf{R}^{m}, A \in \mathbf{R}^{m \times n}(m<n)$ and $A$ has full rank $m$. The dual linear program of (1.1) can be expressed in the following symmetric form

$$
\begin{array}{ll}
\operatorname{minimize} & d^{T} y \\
\text { subject to } & B y=B c \\
& y \geq 0
\end{array}
$$

where $y \in \mathbf{R}^{n}$ is the vector of dual slack variables, $d=A^{T}\left(A A^{T}\right)^{-1} b, B \in \mathbf{R}^{(n-m) \times n}$ has full rank and $A B^{T}=0$ (i.e., the columns of $B^{T}$ form a basis for the null space of $A$ ). This form of the dual was introduced by Todd and Ye in [9].

It is known that the optimality conditions for (1.1) and (1.2) can be written as a $2 n \times 2 n$ nonlinear system with non-negative variables; namely,

$$
F(x, y)=\left(\begin{array}{c}
A x-b \\
B y-B c \\
X Y e
\end{array}\right)=0, \quad(x, y) \geq 0
$$

where $X=\operatorname{diag}(x), Y=\operatorname{diag}(y)$ and $e$ is the $n$-vector of all ones.

The feasibility set of problem (1.3) is defined as

$$
\mathcal{F}=\left\{(x, y): x, y \in \mathbf{R}^{n}, A x=b, B y=B c,(x, y) \geq 0\right\}
$$

A feasible pair $(x, y) \in \mathcal{F}$ is said to be strictly feasible if it is positive. In this work we assume that strictly feasible points exist.

We denote the solution set of Problem (1.3) by

$$
\mathcal{S}=\{(x, y): F(x, y)=0,(x, y) \geq 0\}
$$


It is known that the existence of strictly feasible points implies that $\mathcal{S}$ is bounded (see the proof of Lemma 2.1 in this paper). In addition, if $\mathcal{S}_{x}$ and $\mathcal{S}_{y}$ are the primal and dual solution sets, respectively, then

$$
\mathcal{S}=\mathcal{S}_{x} \times \mathcal{S}_{y}
$$

In this work, we are only concerned with the case when $\mathcal{S}$ is not a singleton set, i.e.,

$$
r i(\mathcal{S}) \neq \emptyset
$$

where $\operatorname{ri}(\mathcal{S})$ is the relative interior of $\mathcal{S}$. In this case, the solution set $\mathcal{S}$ has the following structure (see [1] for a proof): (i) all points in the relative interior satisfy strict complementarity (and all points on the relative boundary do not); (ii) the zero-nonzero pattern of points in the relative interior is invariant. Therefore, for any $\left(x^{*}, y^{*}\right) \in \operatorname{ri}(\mathcal{S})$, the following index sets

$$
I_{x}^{+}=\left\{i: x_{i}^{*}>0,1 \leq i \leq n\right\} \text { and } I_{y}^{+}=\left\{i: y_{i}^{*}>0,1 \leq i \leq n\right\}
$$

are independent of the choice of $\left(x^{*}, y^{*}\right)$. Moreover, by strict complementarity

$$
I_{x}^{+} \bigcup I_{y}^{+}=\{1,2, \ldots, n\} \text { and } I_{x}^{+} \bigcap I_{y}^{+}=\emptyset
$$

Given $w \in \mathbf{R}^{n}$ such that

$$
w>0 \text { and } e^{T} w=n
$$

the $w$-center of the solution set $\mathcal{S}$ is defined as

$$
\left(x^{w}, y^{w}\right)=\arg \max \left\{\psi^{w}(x, y):(x, y) \in \mathcal{S}\right\}
$$

where

$$
\psi^{w}(x, y)=\prod_{i \in I_{x}^{+}} x_{i}^{w_{i}} \prod_{i \in I_{y}^{+}} y_{i}^{w_{i}}
$$

Equivalently, in (1.5) one can replace $\psi^{w}(x, y)$ by its logarithm, i.e.,

$$
\ln \psi^{w}(x, y)=\sum_{i \in I_{x}^{+}} w_{i} \ln x_{i}+\sum_{i \in I_{y}^{+}} w_{i} \ln y_{i}
$$


Obviously, $\left(x^{w}, y^{w}\right) \in \operatorname{ri}(\mathcal{S})$ and $\psi^{w}\left(x^{w}, y^{w}\right)>0$. From the structure of both $\psi^{w}(x, y)$ and $\mathcal{S}$, we see that $x^{w}$ and $y^{w}$ are the $w$-centers of the primal and the dual solution sets, respectively; namely,

$$
x^{w}=\arg \max \left\{\prod_{i \in I_{x}^{+}} x_{i}^{w_{i}}: x \in \mathcal{S}_{x}\right\} \text { and } y^{w}=\arg \max \left\{\prod_{i \in I_{y}^{+}} y_{i}^{w_{i}}: y \in \mathcal{S}_{y}\right\} .
$$

The existence and uniqueness of $w$-center can be established in a standard manner. For more details, see McLinden [4] and Megiddo [5]. In [4] McLinden called $x^{w}$ and $y^{w}$ the strong Pareto optimal elements of $\mathcal{S}_{x}$ and $\mathcal{S}_{y}$, respectively (see Proposition 5).

For $w \in \mathbf{R}^{n}$ satisfying (1.4), the $w$-path of Problem (1.3), parametrized by $\mu$, is defined as

$$
\mathcal{P}^{w}=\left\{\left(x^{w}(\mu), y^{w}(\mu)\right):\left(x^{w}(\mu), y^{w}(\mu)\right) \in \mathcal{F}, X^{w}(\mu) Y^{w}(\mu) e=\mu w, \mu>0\right\},
$$

In particular, the $w$-path corresponding to $w=e$ is called the central path.

The existence, differentiability and limit behavior of the $w$-paths were first studied by McLinden [4] in a general setting, and later by Megiddo [5] for linear programming in particular. The most striking result in McLinden [4] is perhaps Theorem 9. In the case of linear programming, it states that the limit of the $w$-path as $\mu$ converges to zero is the $w$-center of $\mathcal{S}$, namely,

$$
\left(x^{w}, y^{w}\right)=\lim _{\mu \rightarrow 0}\left(x^{w}(\mu), y^{w}(\mu)\right) .
$$

See also Proposition 8.2 in Megiddo [5] and the discussion preceding it.

The primal-dual interior-point algorithms considered in this research can be motivated in several ways, e.g., path-following or potential reduction, but in essence they are all variants of Newton's method. The following generic algorithmic framework includes a majority of existing primal-dual interior-point algorithms.

\section{Algorithm 1 (Generic Primal-Dual Algorithm)}

Given a strictly feasible pair $\left(x^{0}, y^{0}\right)$ and $w \in \mathbf{R}^{n}$ such that $w>0$ and $e^{T} w=n$. For $k=0,1,2, \ldots, d o$

$$
\text { Choose } \sigma^{k} \in[0,1) \text { and set } \mu^{k}=\sigma^{k} \frac{x^{k} y^{k}}{n} \text {. }
$$


(2) Solve the following system for $\left(\Delta x^{k}, \Delta y^{k}\right)$ :

$$
F^{\prime}\left(x^{k}, y^{k}\right)\left(\begin{array}{c}
\Delta x \\
\Delta y
\end{array}\right)=-F\left(x^{k}, y^{k}\right)+\mu^{k}\left(\begin{array}{c}
0 \\
w
\end{array}\right) .
$$

(3) Choose a step-length $\alpha^{k}=\min \left(1, \tau^{k} \hat{\alpha}^{k}\right)$ for $\tau^{k} \in(0,1)$ and

$$
\hat{\alpha}^{k}=\frac{-1}{\min \left(\left(X^{k}\right)^{-1} \Delta x^{k},\left(Y^{k}\right)^{-1} \Delta y^{k}\right)} \text {. }
$$

(4) Form the new iterate

$$
\left(x^{k+1}, y^{k+1}\right)=\left(x^{k}, y^{k}\right)+\alpha^{k}\left(\Delta x^{k}, \Delta y^{k}\right)
$$

The standard choice for $w$ in Algorithm 1 is $w=e$. Since the analysis is no more complicated for general $w$, we will carry out our analysis for the general case.

A straightforward calculation gives

$$
F^{\prime}(x, y)=\left[\begin{array}{cc}
A & 0 \\
0 & B \\
Y & X
\end{array}\right] .
$$

There are two fundamental parameters in Algorithm 1, the centering parameter $\sigma^{k}$ and the step-length $\tau^{k}$ (or $\left.\alpha^{k}\right)$. The choice of step-length $\alpha^{k}$ guarantees $\left(x^{k+1}, y^{k+1}\right)>0$. It is easy to verify that the iterates satisfy the following useful relationships

$$
x^{k+1} y^{k+1}=\left(1-\alpha^{k}\left(1-\sigma^{k}\right)\right) x^{k^{T}} y^{k}
$$

and

$$
Y^{k} \Delta x^{k}+X^{k} \Delta y^{k}=-X^{k} Y^{k} e+\sigma_{k} \frac{x^{k^{T}} y^{k}}{n} w .
$$

The first primal-dual interior-point algorithm for linear programming was constructed by Kojima, Mizuzo and Yoshise [3]. It was based on the idea of following the central path (i.e., $w=e$ ) which was studied in Megiddo [5] (see also Sonnevend [7]). Other early primal-dual interior-point algorithms using the central path include Monteiro and Adler [6] and Todd and Ye [9]. 
The convergence properties of the duality gap sequence have been studied extensively by many authors. On the other hand, the convergence properties of the iteration sequence were investigated only very recently. Tapia, Zhang and Ye [8] showed that under suitable conditions, if $\sigma^{k}$ is chosen to converge to zero fast enough, then the iteration sequence will converge to a solution. At this juncture, it is still unknown if the iteration sequence converges when $\left\{\sigma^{k}\right\}$ does not converge to zero; though in practice convergence seems to be the norm rather than the exception.

Another unanswered question is whether a path-following interior-point algorithm can be constructed with the property that when the iteration sequence converges, it actually converges to the center of the solution set. In the next section, we establish that convergence to the center of the solution set is indeed attainable.

\section{Main Result}

We begin by introducing our notation. Define

$$
\phi_{i}^{k}=x_{i}^{k} y_{i}^{k}, \quad i=1,2, \ldots, n,
$$

and

$$
\bar{\phi}^{k}=\frac{1}{n} \sum_{i=1}^{n} \phi_{i}^{k} .
$$

It follows from (4) of Algorithm 1, (1.11) and (1.12) that

$$
\phi_{i}^{k+1}=\phi_{i}^{k}\left(1-\alpha^{k}\right)+w_{i} \sigma^{k} \bar{\phi}^{k} \alpha^{k}+\Delta x_{i}^{k} \Delta y_{i}^{k}\left(\alpha^{k}\right)^{2}
$$

and

$$
\bar{\phi}^{k+1}=\bar{\phi}^{k}\left(1-\alpha^{k}+\sigma^{k} \alpha^{k}\right) .
$$

Therefore,

$$
\phi_{i}^{k+1}-w_{i} \bar{\phi}^{k+1}=\left(\phi_{i}^{k}-w_{i} \bar{\phi}^{k}\right)\left(1-\alpha^{k}\right)+\Delta x_{i}^{k} \Delta y_{i}^{k}\left(\alpha^{k}\right)^{2} .
$$

This leads to

$$
\frac{\phi_{i}^{k+1}-w_{i} \bar{\phi}^{k+1}}{\bar{\phi}^{k+1}}=\frac{\phi_{i}^{k}-w_{i} \bar{\phi}^{k}}{\bar{\phi}^{k}} \frac{1-\alpha^{k}}{1-\alpha^{k}+\sigma^{k} \alpha^{k}}+\frac{\Delta x_{i}^{k} \Delta y_{i}^{k}}{\bar{\phi}^{k}} \frac{\left(\alpha^{k}\right)^{2}}{1-\alpha^{k}+\sigma^{k} \alpha^{k}} .
$$


Let

$$
\eta^{k}=\max _{1 \leq i \leq n}\left|\frac{\phi_{i}^{k}}{\bar{\phi}^{k}}-w_{i}\right| .
$$

Now, from (2.3) and the fact that $\phi_{i}^{k} / \bar{\phi}^{k} \leq n$ we have

$$
\eta^{k+1} \leq \frac{1-\alpha^{k}}{1-\alpha^{k}+\sigma^{k} \alpha^{k}} \eta^{k}+\max _{1 \leq i \leq n}\left\{\frac{\left|\Delta x_{i}^{k} \Delta y_{i}^{k}\right|}{x_{i}^{k} y_{i}^{k}}\right\} \frac{n\left(\alpha^{k}\right)^{2}}{1-\alpha^{k}+\sigma^{k} \alpha^{k}} .
$$

For simplicity, in what follows we will drop the superscript " $w$ " from the points on the $w$-path, i.e., we will use the convention

$$
(x(\mu), y(\mu)) \equiv\left(x^{w}(\mu), y^{w}(\mu)\right) .
$$

The following lemma states a very intuitive result; yet it is central to the establishment of our main result. A rigorous proof for this lemma is in order.

Lemma 2.1 Let $\left\{\left(x^{k}, y^{k}\right)\right\} \subset \mathcal{F}$. Assume $x^{k^{T}} y^{k} \rightarrow 0$ and $\eta^{k} \rightarrow 0$. Then $\left\{\left(x^{k}, y^{k}\right)\right\}$ converges to the $w$-center of the solution set, i.e.,

$$
\lim _{k \rightarrow \infty}\left(x^{k}, y^{k}\right)=\left(x^{w}, y^{w}\right)
$$

Proof: The assumption $x^{k^{T}} y^{k} \rightarrow 0$ and (1.8) imply that (recall $\bar{\phi}^{k}=\frac{x^{k^{T}} y^{k}}{n}$ )

$$
\lim _{k \rightarrow \infty}\left(x\left(\bar{\phi}^{k}\right), y\left(\bar{\phi}^{k}\right)\right)=\left(x^{w}, y^{w}\right) .
$$

Since

$$
\left\|\left(x^{k}, y^{k}\right)-\left(x^{w}, y^{w}\right)\right\| \leq\left\|\left(x^{k}, y^{k}\right)-\left(x\left(\bar{\phi}^{k}\right), y\left(\bar{\phi}^{k}\right)\right)\right\|+\left\|\left(x\left(\bar{\phi}^{k}\right), y\left(\bar{\phi}^{k}\right)\right)-\left(x^{w}, y^{w}\right)\right\|,
$$

it suffices to prove

$$
\left\|\left(x^{k}, y^{k}\right)-\left(x\left(\bar{\phi}^{k}\right), y\left(\bar{\phi}^{k}\right)\right)\right\| \rightarrow 0 .
$$

Let

$$
\left(\bar{x}^{k}, \bar{y}^{k}\right)=\frac{1}{2}\left(x^{k}+x\left(\bar{\phi}^{k}\right), y^{k}+y\left(\bar{\phi}^{k}\right)\right) .
$$

It can be verified by substitution that

$$
\left[\begin{array}{cc}
A & 0 \\
0 & B \\
\bar{Y}^{k} & \bar{X}^{k}
\end{array}\right]\left(\begin{array}{c}
x^{k}-x\left(\bar{\phi}^{k}\right) \\
y^{k}-y\left(\bar{\phi}^{k}\right)
\end{array}\right)=\left(\begin{array}{c}
0 \\
X^{k} Y^{k} e-\bar{\phi}^{k} w
\end{array}\right)
$$


or

$$
\left(\begin{array}{c}
x^{k}-x\left(\bar{\phi}^{k}\right) \\
y^{k}-y\left(\bar{\phi}^{k}\right)
\end{array}\right)=\left[F^{\prime}\left(\bar{x}^{k}, \bar{y}^{k}\right)\right]^{-1}\left(\begin{array}{c}
0 \\
X^{k} Y^{k} e-\bar{\phi}^{k} w
\end{array}\right) .
$$

For $(x, y)>0$, define

$$
\begin{aligned}
P(x, y) & =I-\left(X Y^{-1}\right)^{1 / 2} A^{T}\left(A X Y^{-1} A^{T}\right)^{-1} A\left(X Y^{-1}\right)^{1 / 2} \\
H_{p}(x, y) & =(X Y)^{-1 / 2} P(x, y)(X Y)^{-1 / 2} \\
H_{d}(x, y) & =(X Y)^{-1 / 2}(I-P(x, y))(X Y)^{-1 / 2}
\end{aligned}
$$

It is worth noting that $P(x, y)$ and $I-P(x, y)$ are orthogonal projection matrices. Now it follows from (2.8) (see Lemma 3.1 in [8], for example) that

$$
\left(\begin{array}{c}
x^{k}-x\left(\bar{\phi}^{k}\right) \\
y^{k}-y\left(\bar{\phi}^{k}\right)
\end{array}\right)=\left(\begin{array}{c}
\bar{X}^{k} H_{p}\left(\bar{x}^{k}, \bar{y}^{k}\right)\left(X^{k} Y^{k} e-\bar{\phi}^{k} w\right) \\
\bar{Y}^{k} H_{d}\left(\bar{x}^{k}, \bar{y}^{k}\right)\left(X^{k} Y^{k} e-\bar{\phi}^{k} w\right)
\end{array}\right)
$$

Therefore, there exists a constant $\beta>0$ such that

$$
\begin{aligned}
\left\|x^{k}-x\left(\bar{\phi}^{k}\right)\right\| & \leq \beta\left\|\bar{x}^{k}\right\|\left\|\bar{\phi}^{k} H_{p}\left(\bar{x}^{k}, \bar{y}^{k}\right)\right\| \eta^{k} \\
\left\|y^{k}-y\left(\bar{\phi}^{k}\right)\right\| & \leq \beta\left\|\bar{y}^{k}\right\|\left\|\bar{\phi}^{k} H_{d}\left(\bar{x}^{k}, \bar{y}^{k}\right)\right\| \eta^{k}
\end{aligned}
$$

Clearly, (2.6) will be proved if we can show that $\bar{\phi}^{k} H_{p}\left(\bar{x}^{k}, \bar{y}^{k}\right), \bar{\phi}^{k} H_{d}\left(\bar{x}^{k}, \bar{y}^{k}\right)$ and $\left(\bar{x}^{k}, \bar{y}^{k}\right)$ are uniformly bounded.

It is known that the level set

$$
\mathcal{L}(\xi)=\mathcal{F} \cap\left\{(x, y): x^{T} y \leq \xi\right\}
$$

is bounded for all $\xi \geq 0$. To see this, observe that given a fixed positive $(\hat{x}, \hat{y}) \in \mathcal{F}$, for any $(x, y) \in \mathcal{L}(\xi)$, we have $(x-\hat{x})^{T}(y-\hat{y})=0$, which leads to

$$
(\hat{y}, \hat{x})^{T}(x, y)=x^{T} y+\hat{x}^{T} \hat{y} \leq \xi+\hat{x}^{T} \hat{y}
$$

This implies the boundedness of $\mathcal{L}(\xi)$. In particular, the solution set $\mathcal{S}=\mathcal{L}(0)$ is bounded.

Using the relation $\left(x^{k}-x\left(\bar{\phi}^{k}\right)\right)^{T}\left(y^{k}-y\left(\bar{\phi}^{k}\right)\right)=0$, we have

$$
y\left(\bar{\phi}^{k}\right)^{T} x^{k}+x\left(\bar{\phi}^{k}\right)^{T} y^{k}=2 x^{k} y^{k}
$$


and hence

$$
\left(\bar{x}^{k}\right)^{T} \bar{y}^{k}=\frac{1}{4}\left(x^{k} y^{k}+y\left(\bar{\phi}^{k}\right)^{T} x^{k}+x\left(\bar{\phi}^{k}\right)^{T} y^{k}+x\left(\bar{\phi}^{k}\right)^{T} y\left(\bar{\phi}^{k}\right)\right)=x^{k T} y^{k} .
$$

Thus $\left(\bar{x}^{k}, \bar{y}^{k}\right) \in \mathcal{L}\left(x^{k^{T}} y^{k}\right) \subset \mathcal{L}\left(x^{0^{T}} y^{0}\right)$. The uniform boundedness of $\left(\bar{x}^{k}, \bar{y}^{k}\right)$ now follows from the boundedness of the level set $\mathcal{L}\left(x^{0} y^{0}\right)$.

To show the uniform boundedness of $\bar{\phi}^{k} H_{p}\left(\bar{x}^{k}, \bar{y}^{k}\right)$, it is sufficient to demonstrate that $\bar{\phi}^{k} /\left(\bar{x}_{i}^{k} \bar{y}_{i}^{k}\right)$ is uniformly bounded for all $i$ because $P\left(\bar{x}^{k}, \bar{y}^{k}\right)$ is an orthogonal projection matrix. In view of the fact that $\left(x^{k}, y^{k}\right)>0$ and $\left(x\left(\bar{\phi}^{k}\right), y\left(\bar{\phi}^{k}\right)\right)>0$, we have for all $i$

$$
\frac{\bar{\phi}^{k}}{\bar{x}_{i}^{k} \bar{y}_{i}^{k}}=\frac{\bar{\phi}^{k}}{\frac{1}{4}\left(x_{i}^{k}+x_{i}\left(\bar{\phi}^{k}\right)\right)\left(y_{i}^{k}+y_{i}\left(\bar{\phi}^{k}\right)\right)} \leq \frac{4 \bar{\phi}^{k}}{x_{i}^{k} y_{i}^{k}+\bar{\phi}^{k} w_{i}}<\frac{4}{\min \left(w_{i}\right)} \text {. }
$$

Similarly, we can show the uniform boundedness of $\bar{\phi}^{k} H_{d}\left(\bar{x}^{k}, \bar{y}^{k}\right)$. This completes the proof.

Now we are ready to state our main result.

Theorem 2.1 Let $\left\{\left(x^{k}, y^{k}\right)\right\}$ be generated by Algorithm 1 with the parameter choices $\left\{\sigma^{k}\right\}$ and $\left\{\alpha^{k}\right\}$. Assume

A1 $\sigma^{k} \geq \sigma$ and $\alpha^{k} \geq \alpha$ for some $\sigma, \alpha \in(0,1)$

A2 $\min \left(X^{k} Y^{k} e\right) /\left(x^{k}\right)^{T} y^{k} \geq \gamma / n$ for some $\gamma \in(0,1)$;

A3 $\left\{\left(x^{k}, y^{k}\right)\right\}$ converges to $\left(x^{*}, y^{*}\right)$.

Then $\left\{\left(x^{k}, y^{k}\right)\right\}$ converges to the $w$-center of the solution set, i.e., $\left(x^{*}, y^{*}\right)=\left(x^{w}, y^{w}\right)$.

Proof: From (1.11), Assumption A1 implies that the duality gap sequence converges to zero. Hence, $\left(x^{*}, y^{*}\right)$ is a solution. In addition, by Güler and Ye [2] Assumption A2 implies that $\left(x^{*}, y^{*}\right)$ satisfies strict complementarity.

By Lemma 2.1, it suffices to prove $\left\{\eta^{k}\right\}$ converges to zero. First we prove that $\left\{\eta^{k}\right\}$ cannot be bounded away from zero. Suppose this is not true, i.e. $\eta^{k} \geq \eta$ for some $\eta>0$. Then we can write (2.5) as

$$
\eta^{k+1} \leq \beta^{k} \eta^{k}
$$


where

$$
\beta^{k}=\frac{1-\alpha^{k}}{1-\alpha^{k}+\sigma^{k} \alpha^{k}}+\max _{1 \leq i \leq n}\left\{\frac{\left|\Delta x_{i}^{k} \Delta y_{i}^{k}\right|}{x_{i}^{k} y_{i}^{k}}\right\} \frac{n\left(\alpha^{k}\right)^{2}}{\eta\left(1-\alpha^{k}+\sigma^{k} \alpha^{k}\right)} .
$$

A contradiction will arise if $\beta^{k} \leq \beta$ for some $\beta \in(0,1)$, which leads to $Q$-linear convergence of $\left\{\eta^{k}\right\}$ to zero.

Under Assumption A1, there exist constants $\beta^{\prime} \in(0,1)$ and $\beta^{\prime \prime}>0$ such that

$$
\frac{1-\alpha^{k}}{1-\alpha^{k}+\sigma^{k} \alpha^{k}} \leq \beta^{\prime}<1
$$

and

$$
\frac{n\left(\alpha^{k}\right)^{2}}{\eta\left(1-\alpha^{k}+\sigma^{k} \alpha^{k}\right)} \leq \beta^{\prime \prime}
$$

Hence, from (2.12) we have

$$
\beta^{k} \leq \beta^{\prime}+\beta^{\prime \prime} \max _{1 \leq i \leq n}\left\{\frac{\left|\Delta x_{i}^{k} \Delta y_{i}^{k}\right|}{x_{i}^{k} y_{i}^{k}}\right\} .
$$

Now consider $x_{i}^{*}>0$. Obviously,

$$
1=\lim _{k \rightarrow \infty} \frac{x_{i}^{k+1}}{x_{i}^{k}}=\lim _{k \rightarrow \infty}\left(1+\alpha_{k} \frac{\Delta x_{i}^{k}}{x_{i}^{k}}\right) .
$$

This implies $\Delta x_{i}^{k} / x_{i}^{k} \rightarrow 0$, because $\left\{\alpha_{k}\right\}$ is bounded away from zero. Moreover, it is wellknown that Assumption A2 implies that $\left|\Delta y_{i}^{k} / y_{i}^{k}\right|$ is uniformly bounded (see [12], for example). On the other hand, if $x_{i}^{*}=0$, then by strict complementarity $y_{i}^{*}>0$. The same argument, interchanging the roles of $x$ and $y$, gives that $\Delta y_{i}^{k} / y_{i}^{k} \rightarrow 0$ and $\left|\Delta x_{i}^{k} / x_{i}^{k}\right|$ is uniformly bounded. Hence

$$
\frac{\Delta x_{i}^{k} \Delta y_{i}^{k}}{x_{i}^{k} y_{i}^{k}} \rightarrow 0, \quad i=1,2, \ldots, n .
$$

Consequently, for $k$ sufficiently large

$$
\max _{1 \leq i \leq n}\left\{\frac{\left|\Delta x_{i}^{k} \Delta y_{i}^{k}\right|}{x_{i}^{k} y_{i}^{k}}\right\} \leq \frac{1-\beta^{\prime}}{2 \beta^{\prime \prime}} .
$$

However, it follows from (2.13) that for $k$ sufficiently large

$$
\beta^{k} \leq \beta=\frac{1+\beta^{\prime}}{2}<1 .
$$


This contradicts the hypothesis $\eta^{k} \geq \eta>0$. Hence $\left\{\eta^{k}\right\}$ cannot be bounded away from zero. As a result, there must exist a subsequence of $\left\{\eta^{k}\right\}$ that converges to zero. Consequently, from Lemma 2.1 the corresponding subsequence of the iteration sequence $\left\{\left(x^{k}, y^{k}\right)\right\}$ converges to the $w$-center of the solution set. But since the iteration sequence itself is convergent, the entire sequence has to converge to the $w$-center of the solution set. This completes the proof.

\section{Concluding Remarks}

Assumptions A1-A2 can be easily achieved. For fixed $\sigma^{k}=\sigma \in(0,1)$, Assumption A2 can be enforced by properly choosing $\alpha^{k}$ and at the same time ensuring that $\left\{\alpha^{k}\right\}$ is bounded away from zero. A particular choice for $\alpha^{k}$ is given in [3], for example.

Let us put Theorem 2.1 in perspective. We have shown, under mild assumptions, that if $\left\{\sigma^{k}\right\}$ is bounded away from zero, then whenever the iteration sequence generated by Algorithm 1 converges, it converges to the center of the solution set. Very recently, Tapia, Zhang and Ye [8] demonstrated, under mild assumptions, that if $\left\{\sigma^{k}\right\}$ converges to zero at least $R$-linearly, then the iteration sequence converges to a point in the relative interior of the solution set. In addition, the rate of the convergence of $\left\{\sigma^{k}\right\}$ to zero is reflected in the rate of convergence of the iteration sequence (see also Zhang and Tapia [11]). It is interesting that at this juncture the price one pays for attempting to obtain convergence to the center of the solution set is the loss of a guarantee for the convergence of the iteration sequence and the loss of a guarantee for a fast convergence rate. We strongly believe that this latter loss is a reality; however, based on our numerical experience, we believe that the former is most

likely not a reality. Hence convergence of the iteration sequence for the case when $\left\{\sigma^{k}\right\}$ is bounded below has become an important open question. 


\section{References}

[1] A. El-Bakry, R. A. Tapia, and Yin Zhang. On the use of indicators in identifying zero variables for interior point methods. Technical Report TR91-15, Dept. Mathematical Sciences, Rice University, 1991.

[2] O. Güler and Y. Ye. Convergence behavior of some interior-point algorithms. Working Paper Series No. 91-4, Dept. of Management Sciences, The University of Iowa, 1991.

[3] M. Kojima, S. Mizuno, and A. Yoshise. A primal-dual interior point method for linear programming. In Nimrod Megiddo, editor, Progress in Mathematical programming, interior-point and related methods, pages 29-47. Springer-Verlag, New York, 1989.

[4] L. McLinden. An analogue of Moreau's proximation theorem, with application to the nonlinear complementarity problem. Pacific J. of Math., 88:101-161, 1980.

[5] N. Megiddo. Pathways to the optimal set in linear programming. In Nimrod Megiddo, editor, Progress in Mathematical programming, interior-point and related methods, pages 131-158. Springer-Verlag, New York, 1989.

[6] R.C. Monteiro and I. Adler. Interior path-following primal-dual algorithms. Part I: linear programming. Math. Prog., 44:27-41, 1989.

[7] G. Sonnevend. An analytic center for polyhedrons and new classes of global algorithms for linear (smooth, convex) programming. In A. Prekopa, editor, Lecture Notes in Control and Inf. Sci., Vol. 84:866-876. Springer, Berlin, 1985.

[8] R. A. Tapia, Y. Zhang and Y. Ye On the convergence of the iteration sequence in primal-dual interior-point methods. Technical Report No. 91-24, Dept. of Mathematical Sciences, Rice University, 1991.

[9] M.J. Todd and Y. Ye. A centered projective algorithm for linear programming. Math. of O.R., 15:508-529,1990. 
[10] Y. Zhang and R. A. Tapia. A quadratically convergent polynomial primal-dual interiorpoint algorithm for linear programming. Technical Report No. 90-40, Dept. of Mathematical Sciences, Rice University, 1990.

[11] Y. Zhang and R. A. Tapia. Superlinear and quadratic convergence of primal-dual interior-point methods for linear programming revisited. Technical Report No. 91-27, Dept. of Mathematical Sciences, Rice University, 1991.

[12] Y. Zhang, R. A. Tapia and J. E. Dennis. On the superlinear and quadratic convergence of primal-dual interior point linear programming algorithms. Technical Report TR90-6, Dept. Mathematical Sciences, Rice University, 1990, to appear in SIAM J. Optimization. 